\title{
STUDYING EVOLUTION OF HYDROTHERMAL ALTERATION MATERIALS IN THE TURRIALBA VOLCANO TROUGH MULTISPECTRAL AND HYPERSPECTRAL IMAGES
}

\author{
J. G. Rejas ${ }^{\text {a,b.* }}$, C. Pothier ${ }^{\mathrm{c}}$, C. Rigotti $^{\mathrm{d}}$, N. Méger ${ }^{\mathrm{e}}, \mathrm{I}_{\text {. Vásquez }}{ }^{\mathrm{f}}$, O.C. Rotunno ${ }^{\mathrm{f}}, \mathrm{J}_{\text {. Bonatti }}{ }^{\mathrm{g}}$ and H. Barbosa $^{\mathrm{h}}$ \\ ${ }^{a}$ National Institute for Aerospace Technology, INTA. Ctra. de Ajalvir km 4 s/n \\ 28850 Torrejón de Ardoz (Spain), rejasaj@inta.es \\ ${ }^{\mathrm{b}}$ Department of Engineering and Land Morphology, Technical University of Madrid, UPM. \\ Ramiro de Maeztu, 7, 28040 Madrid (Spain), juangregorio.rejas@upm.es \\ c Univ Lyon, INSA-Lyon, CNRS, LIRIS, UMR5205, F-69621, Villeurbanne, (France), catherine.pothier@insa-lyon.fr \\ ${ }^{d}$ Univ Lyon, INSA-Lyon, CNRS, INRIA, LIRIS, UMR5205, F-69621, Villeurbanne, (France), christophe.rigotti@insa-lyon.fr \\ e Université Savoie Mont Blanc, Polytech Annecy-Chambéry, LISTIC, B.P. 80439, Annecy-le-Vieux, F-74944 Annecy Cedex, \\ nicolas.meger@univ-smb.fr (France) \\ ${ }^{\mathrm{f}}$ Universidade Federal do Rio de Janeiro, COPPE, Cidade Universitária, Rio de Janeiro (Brazil), iselavp@gmail.com, \\ ottorotunno@gmail.com \\ g Costa Rica University, UCR. Campus UCR 4058 San José (Costa Rica), jbonatti2011@ gmail.com \\ ${ }^{\mathrm{h}}$ Universidade Federal de Alagoas-UFAL, Campus AC Simões, Maceió (Brazil), barbosa33@ gmail.com
}

\section{Commission II/III}

KEY WORDS: Hydrothermal Alteration Materials, Time Series, Anomaly Detection (AD), Copernicus, Turrialba Volcano.

\begin{abstract}
:
The aim of this work is to develop a geospatial methodology for the analysis of the time evolution of The Turrialba volcano using different automatic imaging techniques compared to expert-based remote sensing techniques. Change detection of hydrothermal alteration materials in relation with time series from multisensor data acquired in spectral ranges of the visible (VIS) and short wave infrared (SWIR) have been calculated. We used for this purpose multispectral and hyperspectral scenes of the Sentinel 2, ALI and Hyperion sensors, respectively, on four dates from 2013 and 2018. This work adopts a multi-source approach, applied to the analysis of the correlations between hydrothermal materials and spectral anomalies in The Turrialba volcano complex, located in The Central Volcanic Range (Costa Rica).
\end{abstract}

An expert-based technique called Crosta's technique for detecting hydrothermal materials have been applied. We have chosen four variables for generating a different Principal Component Analysis (PCA) for groups of channels, two highly reflective and two highly absorptive for each mineral. We have tested another technique to detect hydrothermal materials based on a discrete spectral profile analysis and an unsupervised data mining approach. In other sense, we have applied an automatic technique called anomaly detection to compare with the hydrothermal materials results. Results are presented as an approach based on a comparison of two different strategies whose main future interest lies in the automated identification of patterns of hydrothermally altered materials without prior knowledge or poor information about the area, which has relevant implications in image-based prospecting.

\section{INTRODUCTION}

Multispectral and hyperspectral imagery has been used in the last decades with satisfactory scientific results to study and monitor a huge number of different applications in the biosphere, geosphere, cryosphere and hydrosphere such as deforestation, fires, volcanic activities, landslides, earthquakes, environment, pollution, sea ice extent, soil moisture or flooding. Data and remote sensing technologies have been improving exponentially, in such a way that they provide information about the Earth with many details and high frequency. The European Earth Observation Program Copernicus has been specially developed for this purpose, one of its main tools being the Sentinel 2 twin satellites (Baillarin et al., 2012), forming a multispectral system of high resolution, both geometric/radiometric and temporal.

Imaging spectrometry, one of the most important imaging techniques, has proven in particular to be effective in the characterization of minerals based on statistical methods using specific reflection and absorption bands. Multispectral configurations in SWIR and VNIR (Visible-Near Infrared) have been successfully used to map hydrothermal alteration materials in different geological scenarios (Antón-Pacheco et al., 2001; Cipar et al., 2011; Crosta et al., 2003). There are some minerals that indicate hydrothermal alteration that we can detect remotely by their spectral responses in the diagnostic absorption and reflection bands. These specific spectral features can be extracted from properly calibrated remote sensing data Argillaceous minerals, such as kaolinite, illite and alunite, have a concrete spectral characteristic presenting a high reflectance between the wavelengths of $1.55 \mu \mathrm{m}$ and $1.75 \mu \mathrm{m}$, they also present high absorption between $2,08 \mu \mathrm{m}$ and 2,35 $\mu \mathrm{m}$. Another characteristic of these minerals is that the rocks that have not suffered the hydrothermal process, usually present regular values for the previously mentioned wave lengths. In addition, the minerals with high content in $\mathrm{Fe}$ present a very high contrast between the wavelengths $0,63 \mu \mathrm{m}$ and $0,69 \mu \mathrm{m}$ and the wavelengths $0,45 \mu \mathrm{m}$ to $0,52 \mu \mathrm{m}$.

* Corresponding author 
In this research, we also rely on Anomaly Detection (AD), been developed in the last decades, allowing a better understanding of a domain that has the sub-pixel differentiation of the spectral mixture and its implications in anomalous responses (Duran and Petrou, 2007; Manolakis and Shaw, 2002; Malpica et al., 2008; Shaum, 2005).

Due to the increasing activity of this complex that requires special attention for its proximity to main urban areas, an important aim in this research is to establish relationships to link spectral patterns to surface phenomena in the area. Results are presented based on two different strategies. The main future interest of the approach in the automated identification of patterns of hydrothermally altered materials without prior knowledge or poor information about the area, which has relevant implications in image-based prospecting.

\section{DATA AND PRE-PROCESSING}

We used multispectral and hyperspectral scenes of the Sentinel 2, ALI and Hyperion sensors, respectively, in a period between 2013, 2017 and 2018. This work implies a multi-source approach, applied to the analysis of the correlations between hydrothermal materials and spectral anomalies in The Turrialba volcano complex, located in The Central Volcanic Range (Costa Rica).

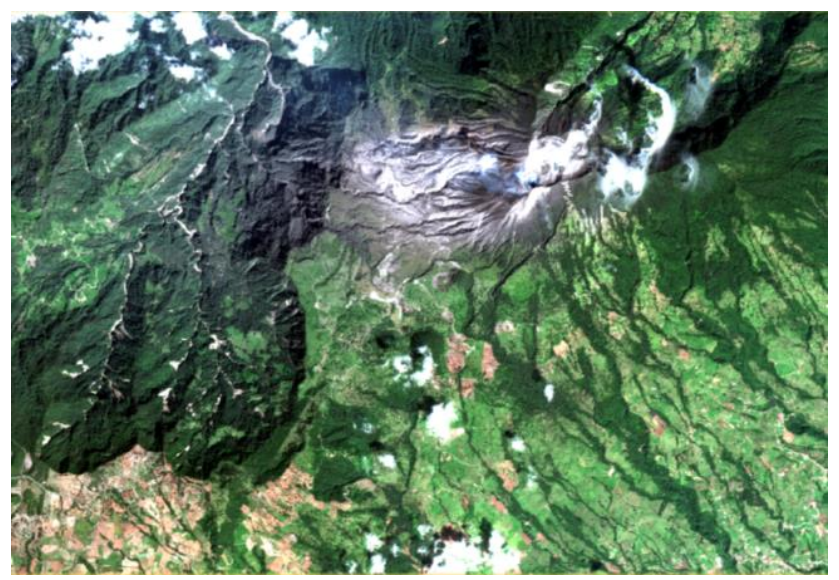

Figure 1. Sentinel 2 natural colour combination RGB 4,3,2 for Turrialba volcano area, 26/01/2017.

Data pre-processing techniques to extract information from all images have been applied. They include geometric correction, radiometric correction and a number of image arithmetic and statistical analysis. This previous step allowed to obtain a perfectly overlapped image data set, the identification of spectral diagnostic bands of hydrothermal alteration minerals and the spatial correlation accuracy.

\section{EVOLUTION OF HYDROTHERMAL ALTERATION IN THE TURRIALBA VOLCANO}

\subsection{Principal component analysis to detect hydrothermal alteration materials}

There are some minerals that indicate hydrothermal alteration that we can detect remotely by their spectral responses in the diagnostic absorption and reflection bands. These specific spectral features can be extracted from the remote sensing data that are properly calibrated.
Argillaceous minerals, such as kaolinite, illite and alunite, have a concrete spectral characteristic presenting a high reflectance between the wavelengths of $1.55 \mu \mathrm{m}$ and $1.75 \mu \mathrm{m}$, they also present high absorption between $2,08 \mu \mathrm{m}$ and 2,35 $\mu \mathrm{m}$. Another additional characteristic of those minerals is that the rocks that have not suffered the hydrothermal process, usually present regular values on the wave lengths previously mentioned. The minerals with high content in Fe present a very high contrast between the wavelengths $0,63 \mu \mathrm{m}$ and $0,69 \mu \mathrm{m}$ and the wavelengths $0,45 \mu \mathrm{m}$ to $0,52 \mu \mathrm{m}$.

An expert-based technique called Crosta's technique for detecting hydrothermal materials have been applied. We have chosen four variables for generating a different Principal Component Analysis (PCA) for groups of channels, two highly reflective and two highly absorptive for each mineral (Crosta $e t$ al., 2004; Rejas et al., 2012).

For Sentinel 2 the channels used to analyse the clay were 2, 8, 11 and 12, while for ALI the channels were 3,7,9 and 10, and for Hyperion after radiometric correction the channels were 14, 48, 150 and 206. We also defined diagnostic bands following the same criteria for a group of $\mathrm{Fe}$ minerals, and for this purpose the channels used of Sentinel 2 were 2, 4, 8 and 13, for ALI the channels were 3, 5, 6 and 9, and for Hyperion the channels $14,31,48$ and 151.

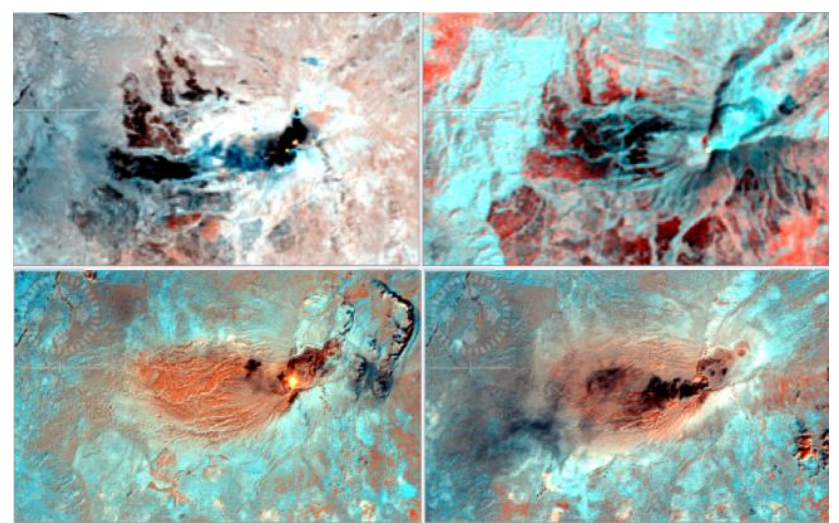

Figure 2. Crosta's technique RGB combination $\mathrm{Ar}, \mathrm{Ar}+\mathrm{Fe}, \mathrm{Fe}$ for Turrialba volcano area in 07/04/2013 (left up ALI), 05/09/2013 (right up Hyperion), 26/01/2017 (left down Sentinel 2) and 12/12/2018 (right down Sentinel 2).

These images came from a principal component analysis and included three bands, one band where the clays were concentrated (Red, R), another band where the iron oxides were concentrated (Blue, B) and another band that was a sum of clays and iron oxides (green band, G). An increase on the evolution of the volcano activity between 2013 (Figure 2 up) and 2017 (Figure 2 left down) and 2018 (Figure 2 right down) is observed.

The subtraction of the clay bands plus iron oxides between the different dates, adopting 2017 as a reference, have been calculated. Once the statistics of the differences were calculated, we verified that the new variables fit the Gaussian bell, as expected.

Everything out of -3-sigma and +3 -sigma are the abnormal changes in the hydrothermal alteration material concentrations, the most interesting changes. In our case, it is observed that they appear in the crater of the Turrialba volcano and on the 
South-West slope, in addition to some cultivation plots in the South of the volcano (see Figure 3).

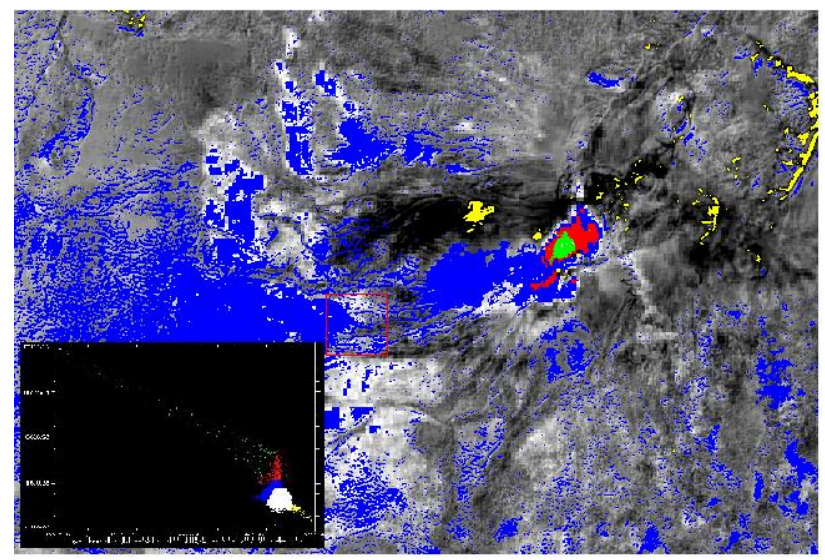

Figure 3. Scatter plot between Ar+Fe between the differences for 2013 and 2018 taking 2017 as a reference (in green 3-sigma, red 2-sigma, blue 1-sigma, yellow 3+sigma).

\subsection{Discrete spectral profile analysis}

We have tested another technique to detect hydrothermal materials based on an unsupervised data mining approach. It uses the quantized spectra of the pixels of the multispectral/hyperspectral images, and in these discrete spectral profiles it finds local shapes that are shared by a significant number of pixels. In addition, it retains a shape only if it is coherent over space, meaning that if the shape is found in the spectral profile of a pixel, then it tends to occur also in the spectral profiles of the adjacent pixels. Let us consider the spectral profiles over the 13 channels of Sentinel 2, after quantization, a discrete spectral profile for a pixel can be, for instance, "1, 2, 2, 2, 2, 3, 3, 3, 3, 3, 3, 2, 2", where the intensity in each channel has been encoded using three symbols: 1 (low), 2 (medium) and 3 (high). A local shape is a sequence of consecutive symbols in this discrete spectral profile, as for example, "2, 2, 3, 3, 3, 3, 3, 3, 2".

Among all possible shapes, a local shape is retained only if it occurs in at least $\mathrm{S} \%$ of the pixels of the image (a minimum coverage constraint) and if when it occurs in a profile then, on average, it also occurs in $\mathrm{K}$ of the 8 neighbouring pixels (a minimum average connectivity constraint). Thus, a selected local shape denotes a regularity in the spectral profiles occurring in a coherent way over space.

The efficient extraction of these local shapes is based on a pattern mining technique (Méger et al. 2019) adapted here to handle spectral profiles. Examples of shapes that can be retrieved from these profiles, include (but are not limited to) plateau (flat zone), peak, valley. Indeed, the kinds of shapes are not predefined and can be of various lengths (i.e., shapes covering different numbers of channels), the only syntactic constraint is that a shape must range over channels that are adjacent in the spectra.

Each selected local shape is then visualized using an "occurrence map" where highlighted pixels represent the pixels containing the local shape in their spectral profile. For a given local shape, comparing its occurrence maps at two different dates provides insights about the evolution in the area. This is illustrated by Figure 4, showing the occurrence maps of two local shapes extracted in an unsupervised way from two Sentinel 2 images.

The first image is the one given Figure $1(26 / 01 / 2017)$ and the second is an acquisition over the same area on 12/12/2018. The occurrence maps (left up, right up) are the ones obtained for the local shape $3,3,3,3,3,3,3,3,3,3,3,3,3$ ranging from channels 1 to 13 , where the symbol 3 denotes high intensity in these channels.

These maps correspond to clouds in the images (26/01/2017 left up, 12/12/2018 right up). Another local shape obtained from the discrete spectral profiles in these images is 2, 3, 3, 3, 3, 2, 1, 2 ranging from channels 5 to 12 , and its occurrence maps (26/01/2017 left down, 12/12/2018 right down) correspond to vegetation that do not change between the two dates (trees along brooks). These local shapes where obtained for $\mathrm{S}=1 \%$ and $\mathrm{K}=4$.
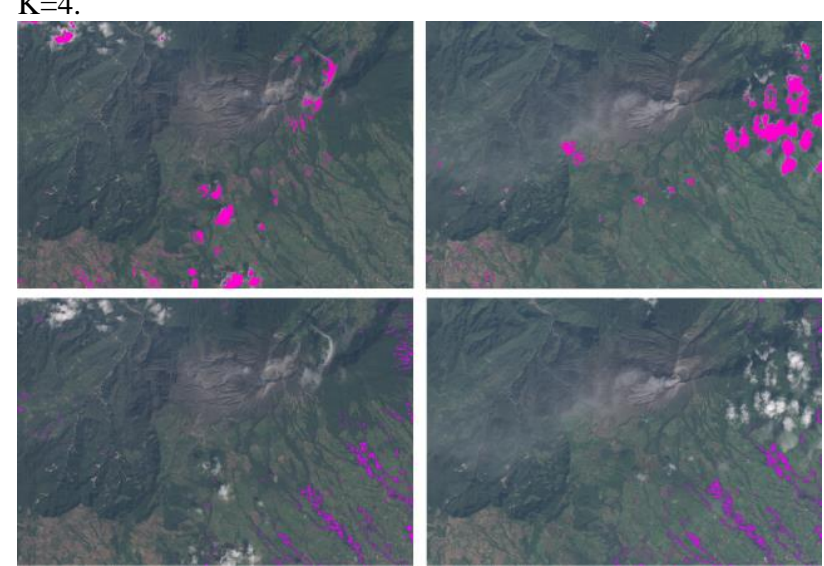

Figure 4. Example of occurrences maps of local shapes found in the discrete spectral profiles of two Sentinel 2 images. Maps highlighting clouds on 26/01/2017 (left up) and on 12/12/2018 (right up). Maps highlighting trees along brooks on 26/01/2017 (left down) and on 12/12/2018 (right down).

\subsection{Anomaly Detection}

Detection of spectral anomalies aims at extracting automatically pixels that show significant responses in relation of their neighbourhood. Several methods have been developed in the last decades, allowing a better understanding of the relationships between spectral data dimensionality and the optimization of search procedures as well as the sub-pixel differentiation of the spectral mixture and its implications in anomalous responses (Duran and Petrou, 2007; Manolakis and Shaw, 2002; Malpica et al., 2008; Shaum, 2005).

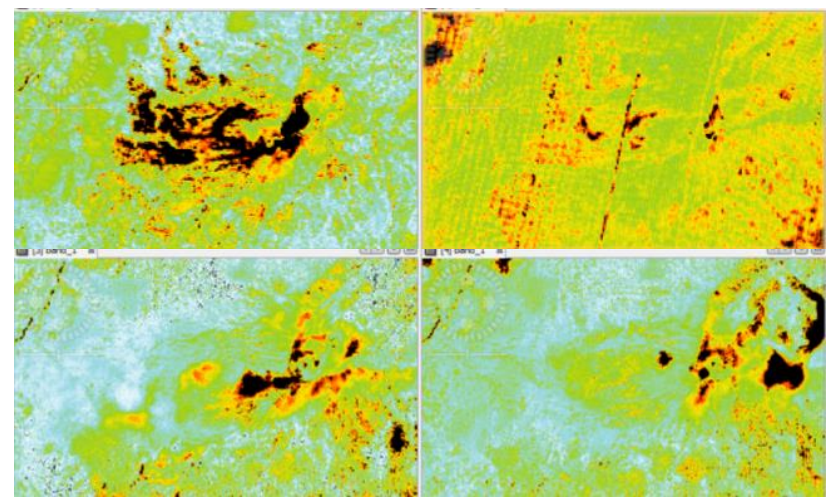

Figure 5. RX spectral anomalies obtained from images in 07/04/2013 (left up ALI), 05/09/2013 (right up Hyperion), 
26/01/2017 (left down Sentinel 2) and 12/12/2018 (right down Sentinel 2). High anomalies in black, the big anomaly areas corresponding to clouds.

A RX algorithm (Reed and Xaoli, 1996), widely accepted as a standard spectral anomaly detection, has been applied to all set of data. Higher concentrations of hydrothermal alteration minerals, in scenarios where the sources of error (mainly vegetation and clouds) are minimized, are correlated with the anomalies calculated in the spectral range reflective for Sentinel 2 and ALI. Spectral anomalies computed from Hyperion data mainly show the high signal-to-noise ratio presented in this particular scene.

Anomaly detection results for ALI, Hyperion and Sentinel 2 sensors are similar, but we have observed the effect of a pattern due to the acquisition process in the Hyperion case (Figure 5, right up). High anomalies areas corresponding to clouds in this tropical spectral background. However, the main anomalies detected in relation with this study are those corresponding to the hydrothermal alteration pixels detected by the others image methods.

The correlation between RXD spectral anomalies and hydrothermal alteration, clays plus iron oxides have been carried out. In order to do that, it has been linearly adjusted, a sample space of 30 pairs of points, placing spectral anomalies in the $\mathrm{Y}$ axis and altered minerals in the $\mathrm{X}$ axis.

Independent samples of hydrothermal alteration and RX spectral anomalies have been statistically compared. Both samples have standardized kurtosis values outside the normal range. In the case of hydrothermal alteration, the check points corresponding to clouds are eliminated, the sample is adjusted to normality. While the non-normality of spectral anomalies is expected in a tropical scenario due to the anomalous response depends on the relationship between the spectral background and the target.

All regressions have been calculated at a confidence level of $95 \%$, removing in each adjustment, the sample values that showed unusual residues and which correspond mainly with clouds and shadow. The results obtained are summarized in Table 1 .

\begin{tabular}{|l|l|l|l|}
\hline Models $\mathrm{AD}$ vs $H T$ & $P$-value & Correlation & $R^{2}(\%)$ \\
\hline $\mathrm{AD}_{\mathrm{S} 22017}=28,2304-0,0633241 * \mathrm{HT}_{\mathrm{S} 22017}$ & 0,1857 & $-0,7216$ & 58,08 \\
\hline $\mathrm{AD}_{\mathrm{S} 22018}=24,9893-0,0432465 * \mathrm{HT}_{\mathrm{S} 22018}$ & 0,2921 & $-0,5456$ & 29,77 \\
\hline $\mathrm{AD}_{\mathrm{ALL} 2013}=13,6052-0,0767031 * \mathrm{HT}_{\mathrm{ALL} 2013}$ & 0,0852 & $-0,9181$ & 84,29 \\
\hline
\end{tabular}

Table 1. Comparison of relationships between spectral Anomalies (AD) and hydrothermal alteration (HT) for Sentinel 2 images of 2017 and 2018, and for ALI image of 2013.

For example, for 2017 (see Figure 6), since the P-value in the ANOVA table is less than 0.05 , there is a statistically relationship significant between anomaly detection and $\mathrm{Ar}+$ Fe_hydrothermal alteration with a confidence level of $95.0 \%$. The R-Square statistic indicates that the adjusted model explains $52.0811 \%$ of the variability in Anomaly Detection.

The mean absolute error (MAE) of 20.7276 is the average value of the residuals. The residuals were examined to determine if there is any significant correlation based on the order in which it is presented in the data file. Since the P-value is greater than
0.05 , there is no indication of a serial autocorrelation in the residuals with a confidence level of $95.0 \%$.

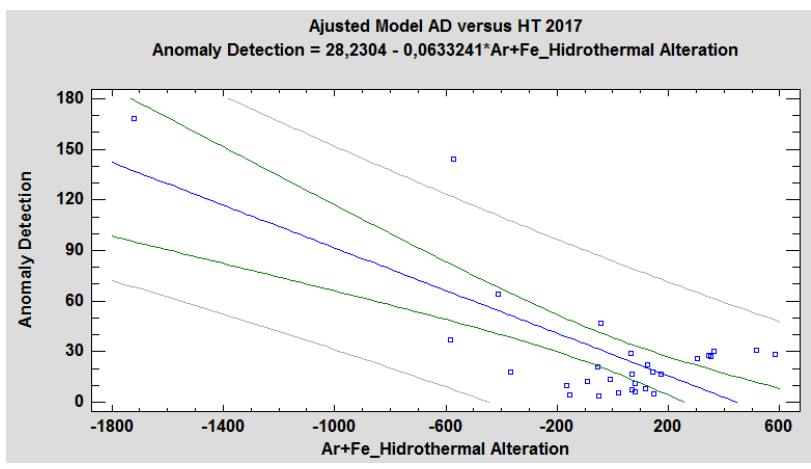

Figure 6. Correlation fit model example Anomaly Detection vs Hydrothermal Alteration for 2017.

\section{VALIDATION AND DISCUSSION}

A check of radiometric and geometric corrections of images have been performed from data measured in supervision campaigns. The selected training areas have been visited and for each one of them, the type of material has been identified. In August 2017 and July 2018 has been carried out a field survey, which allowed us samples of 29 and 30 points respectively, measured in field and laboratory with USB400 and ASD FieldSpec 4 Hi-Res spectroradiometers.

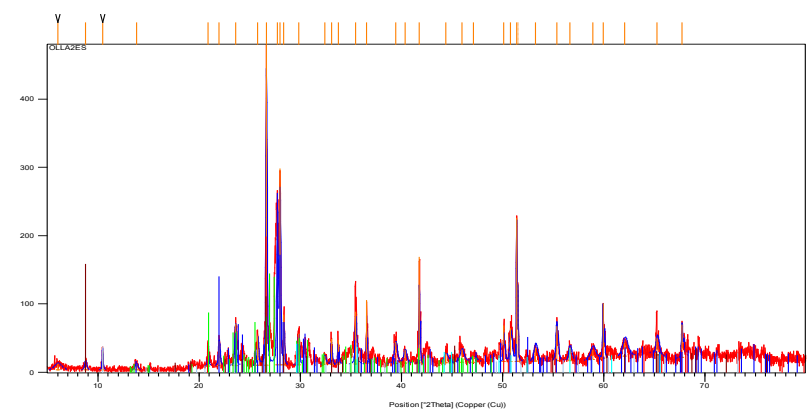

Figure 7. Mineral composition example for the 5 check point in the Turrialba volcano, 2013.

The spectra from the laboratory and the ground truth data have been directly compared with the reflection apparent data on the sensor. This process has allowed to detect the spectral pure pixel in the training areas. The spectra have been used to characterize hydrothermal alteration materials and to check Sentinel 2 reflectance images by empirical linear regression.

Hydrothermal alteration for all images have been classified (see Figure 7) with an unsupervised K-Means algorithm in six clusters. It should be noted that the maps were obtained by unsupervised classification of hydrothermal alteration Crosta's images. Therefore, the result should be understood as a map with six classes supposed to correspond to six degrees of hydrothermal alteration.

The next step we have taken is to make a correspondence between the information of these groups with the real information of the use of the land for 2017 and 2018. For this validation we used data from the two years, in addition to our fieldwork experience in the field. 
Well, therefore, the conclusion is that in Sentinel 2 maps of hydrothermal alteration in 2017 and 2018, group 4 corresponds in both cases to the highest concentration of hydrothermal materials, clays and iron oxides. The other groups are correlated with a mixture of vegetation cover (vegetation with different phenological states, water, clouds, shadows and certain artificial materials).

Note that these are not land cover maps. In this sense, the hydrothermal alteration maps would correspond (with an inherent error obviously) to a certain ground cover as follows: green-lush vegetation; light blue-vegetation; dark bluevegetation affected by acid rain; yellow-crop fields in medium or high phenological state, corpses; red-hydrothermal alteration materials, clays and iron oxides, and sum of clays and iron oxides; white-clouds and fumaroles, that are the amount of gases released into the atmosphere as a result of the volcano's activity.

The most relevant thing is to identify the group in red as the one with the highest concentration of hydrothermal materials. The rest of the colors and groups contains a lot of mismatches with respect to the ground cover.

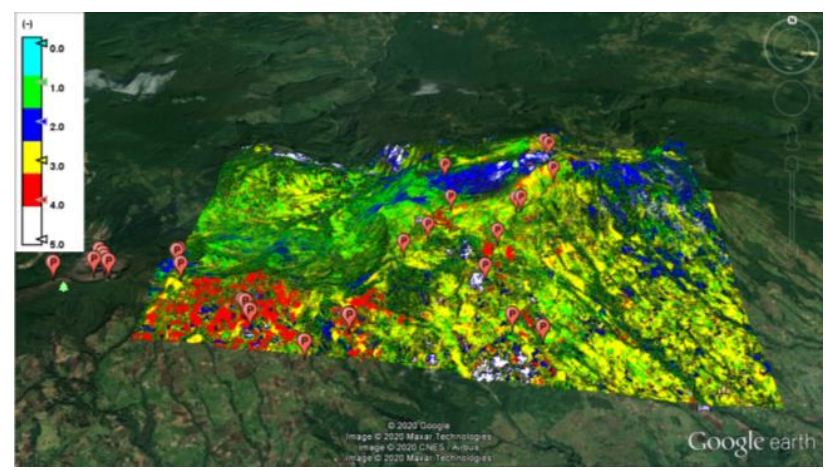

Figure 8. Validation points in the Turrialba volcano for 2017 and 2018 .

Hydrothermal alteration detected from Sentinel 2 in Turrialba and environment, is linked to crops and bare areas, as well as to artificial surfaces (roofs of buildings mainly). This does not mean that in the vicinity of these areas there are no hydrothermal alteration minerals. It can be observed that the detection is strongly influenced by the contribution of the broad, dense and homogeneous natural vegetation cover existing on the spectral information recorded by space sensors.

The evaluation of the hydrothermal alteration detection for Sentinel 2 in 2017 and 2018 images was performed with the method of SNAP programme. The Crosta's technique applied on the classification of this area for 6 cluster presented satisfactory results. Nine of the twelve validation points of hydrothermal alteration for 2017 are satisfactorily labelled such as hydrothermal alteration $(75 \%)$. Eight of the nine validation points of hydrothermal alteration for 2018 are satisfactorily labelled such as hydrothermal alteration (88\%). Dense vegetation is confusing with low vegetation and crops, and gullies are confusing with vegetation affected by acid rain.

The discrete spectral profile analysis presented Section 3.2, led to occurrence maps coherent with the clusters obtained on the Sentinel 2 images in 2017 (see Figure 8) and 2018. With parameters $\mathrm{S}=1 \%$ (minimum surface coverage) and $\mathrm{K}=4$ (minimum average connectivity constraint) the technique selected 50 (resp. 48) local shapes from the spectral profiles in the 26/01/2017 (resp.12/12/2018) image.

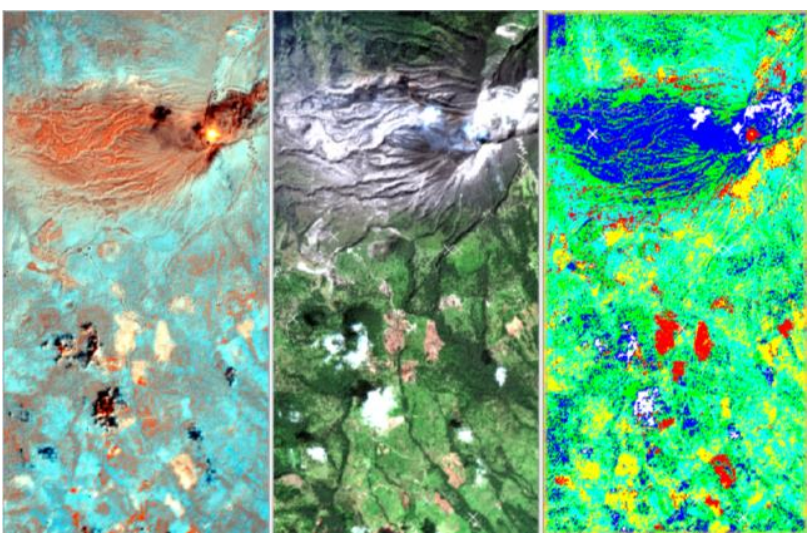

Figure 9. Check and test points for hydrothermal alteration (in red of K-means, right) for Sentinel 22017.

The occurrence maps provide additional evidences of the pertinence of the clusters. For instance, the maps given Figure 10 (2017 left up and 2018 right up) correspond to the red cluster (hydrothermal alteration, see Figure 9 for 2017) in the bottom left corner and is coherent with the evolution of this cluster in this area. In the more central part of the image (south-west flank of the volcano), the maps highlight parts of the red and yellow clusters and again follow their evolution from 2017 to 2018. These maps are those of the local shape 3, 3, 2, 2, 2, 2, 2, 3, 3, 3 ranging from channels 4 to 13 , where symbol 3 (resp. 2) denotes high (resp. medium) intensity in the channels.
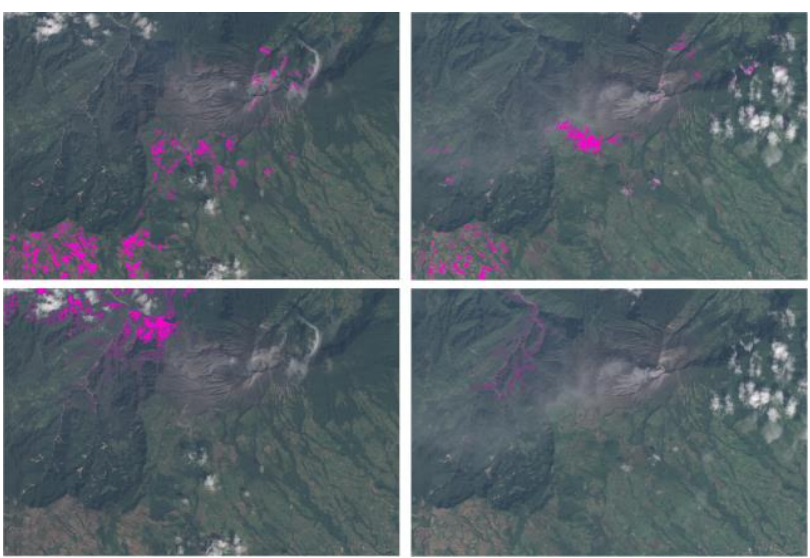

Figure 10. Occurrence maps of local shapes found in the discrete spectral profiles of the two Sentinel 2 images. Maps highlighting parts of clusters red and yellow on 26/01/2017 (left up) and on 12/12/2018 (right up). Maps highlighting parts of cluster dark blue on 26/01/2017 (left down) and on 12/12/2018 (right down).

This local shape, in the spectral profiles, is consistent with the spectral signatures of $\mathrm{Ar}+\mathrm{Fe}$ hydrothermal alterations. A second pair of maps is given Figure 10 (2017 left down and 2018 right down). They correspond to local shape $3,2,1,1,1$, $1,1,1,1,1,1,1,1$ ranging from channels 1 to 13 , and are coherent with the evolution of the upper right part of the dark blue cluster (vegetation affected by acid rain and gullies).

\section{CONCLUSIONS}

The characteristic of high resolution data, both spatial and spectral, for natural covers has been studied by different remote 
detection methods using ALI, Hyperion and Sentinel 2 test data sets in 2013, 2017 and 2018 dates. This paper evaluates the performance of detection methods of hydrothermal alteration materials in scenes with similar backgrounds in the Turrialba volcano (Costa Rica) and it contains a comparison of two different strategies whose main future interest lies in the automated identification of patterns of hydrothermally altered materials without prior knowledge or poor information about the area. The two strategies lead to convergent results that exhibit hydrothermal alteration patterns that are coherent in space and also in their evolution over time.

Higher concentrations of some hydrothermal alteration materials in bared areas where the sources of error are minimized, mainly clouds and vegetation, are correlated with the spectral anomalies in the VNIR range. That is an interesting point because it might be possible to identify some particular spectral bands to carry on monitoring and also detect their environmental impact at the same time.

The spectral mixing associated directly with the spatial resolution has an impact in a significant way on the characterization of the tropical volcanic backgrounds, and thus in the detection of hydrothermal alteration materials used to monitor the dangerous consequence of the volcano activity.

\section{ACKNOWLEDGEMENTS}

The authors would like to acknowledge to the ESA (European Space Agency), NASA (National Aeronautics and Space Administration) and USGS (United States Geological Survey) for providing the data and test data set of Turrialba volcano used to this study.

The present study has been partially supported by the research groups: GIMCC-Grupo de Investigación Mesoamericano en Cambio Climático (Mesomaerican Research Group on Climate Change) and SECFT-Sociedad Española de Cartografía, Fotogrametría y Teledetección (Spanish Society for Cartography, Photogrammetry and Remote Sensing).

Catherine Pothier and Christophe Rigotti are members of LabEx IMU (ANR-10-LABX-0088) that provided complementary support.

\section{REFERENCES}

Antón-Pacheco, C., Rowan, L.C., Mars, J.C. and Gumiel, J.C., 2001. Characterization of mine materials and hydrothermally altered rocks in the Río Tinto mining district (southwest Spain) using HyMAP data. Revista de Teledetección, 2001. Number 16: $65-68$

Baillarin, S. \& Meygret, A. \& Dechoz, C. \& Petrucci, B. \& Lacherade, Sophie \& Tremas, T. \& Isola, C. \& Martimort, Philippe \& Spoto, Francois, 2012. Sentinel-2 Level 1 products and image processing performances. ISPRS - International Archives of the Photogrammetry, Remote Sensing and Spatial Information Sciences. XXXIX-B1. 197-202. 10.5194/isprsarchives-XXXIX-B1-197-2012.

Cipar, J., Anderson, G. and Cooley, T., 2011. Active volcano monitoring using a space-based short-wave infrared imager. Proceedings WHISPERS 2011, Lisbon (Portugal) 6-9 June 2011.
Crosta, A. P., Filho, C. R. de Souza, Azevedo, F. and Brodie, C., 2003. Targeting key alteration minerals in epithermal deposit in Patagonia, Argentina, using ASTER imagery and principal component analysis. International Journal of Remote Sensing, 10, pp. 4233-4240.

Duran, O. and Petrou, M., 2007. A time-efficient method for anomaly detection in hyperspectral images. IEEE Transactions on Geoscience and Remote Sensing, Vol. 45, No. 12, December 2007.

Manolakis, D. and Shaw, G., 2002. Detection Algorithms for Hyperspectral Imaging Applications. IEEE Signal Processing Magazine, January 2002.

Malpica, J.A., Rejas, J.G. and Alonso, M-C., 2008. A projection pursuit algorithm for anomaly detection in hyperspectral imagery. Pattern Recognition 41 (11) pp: 3313-3327, 2008. ISSN 0031-3203.

Méger, N., Rigotti, C., Pothier, C., Nguyen, T., Lodge, F., Gueguen, L., Andréoli, R., Doin, M.-P., and Datcu, M., 2019. Ranking evolution maps for Satellite Image Time Series exploration: application to crustal deformation and environmental monitoring. Data Mining and Knowledge Discovery, Springer, 2019, 33 (1), pp.131-167.

Reed, I.S. and Xiaoli, Y., 1990. Adaptive multiple-band CFAR detection of an optical pattern with unknown spectral distribution. IEEE Transactions on Acoustics. Speech and Signal Processing, Vol. 38, No 10, October 1990.

Rejas, J. G., Martínez-Frías, J., Bonatti, J., Martínez, R., and Marchamalo, M., 2012. Anomaly detection and comparative analysis of hydrothermal alteration materials trough hyperspectral multisensor data in the Turrialba volcano Int. Arch. Photogramm. Remote Sens. Spatial Inf. Sci., XXXIX-B7, 151-155.

Schaum, A., 2005. Hyperspectral detection algorithms: Operational, next generation, on the horizon. Proceedings of the 34th Applied Imagery and Pattern Recognition Workshop (AIPR05).

http://earthexplorer.usgs.gov/

https://scihub.copernicus.eu/dhus/\#/home 